\title{
Incidencias de la educación física y el deporte en la conducta
} psicológica de escolares

\author{
Mirtha Eloísa Díaz de Ríos \\ mirthadiaz2008@hotmail.es \\ Universidad Nacional de Pilar \\ Facultad de Humanidades y Ciencias de la Educación
}

\section{RESUMEN}

El objetivo general del trabajo es analizar la incidencia de la práctica sistemática y metódica de la Educación física y el deporte, en la conducta psicológica de escolares Caso alumnos del $1^{\circ}$ al $6^{\circ}$ grado de la Escuela Básica $N^{\circ} 579$ Defensores del Chaco del Barrio San Lorenzo, año 2021. La presente investigación es del tipo descriptiva, en la que se especificó propiedades, características y rasgos importantes del fenómeno que se analizó, midiendo de manera independiente los conceptos variables a lo que se refiere. Los Resultados señalan que la actividad física (AF) en la escuela posee un rol esencial para la promoción de la actividad física, las preferencias de las actividades físicas ya dependerán de cada niño y niña, pero todas ellas los estarán beneficiando. Para la comprensión de procesos motivacionales, determinante en las conductas psicológicas de escolares saludables de los niños y niñas, ha sido la teoría social-cognitiva, el modelo cognitivo-social está construido sobre expectativas y valores que los alumnos adjudican a diferentes metas y actividades de ejecución.

Palabras Claves: Educación Física; Actividad Física (AF); Conducta de escolares; Incidencia de la Actividad Física. 


\title{
Incidents of Physical Education and Sports in the psychological behavior of schoolchildren
}

\begin{abstract}
The general objective of the work is to analyze the incidence of the systematic and methodical practice of Physical Education and sport, in the psychological behavior of schoolchildren - Case of students from 1st to 6th grade of the Basic School No. 579 Defensores del Chaco del Chaco Barrio San Lorenzo, year 2021. The present investigation is of the descriptive type, in which important properties, characteristics and features of the phenomenon that were analyzed were specified, independently measuring the variable concepts to which it refers. The Results indicate that physical activity (PA) at school plays an essential role in promoting physical activity, the preferences of physical activities will already depend on each boy and girl, but all of them will be benefiting them. For the understanding of motivational processes, a determinant in the psychological behaviors of healthy schoolchildren of boys and girls, has been the social-cognitive theory, the cognitive-social model is built on expectations and values that students assign to different goals and activities of execution.
\end{abstract}

Keywords: Physical Education; Physical Activity (PA); School behavior; Incidence of Physical Activity

Artículo recibido: 03 nov. 2020 Aceptado para publicación: 07 dic. 2020 Correspondencia mirthadiaz2008@ hotmail.es Conflictos de Interés: Ninguna que declarar 


\section{INTRODUCCIÓN}

La Educación Física y el deporte han estado estrechamente vinculados a la salud desde sus inicios como materia en los currículos de las instituciones educativas. Esta relación se ha incrementado en los últimos años a consecuencia de las transformaciones de las sociedades desarrolladas a lo largo del tiempo. Los problemas de salud han cambiado mucho los últimos años, ya no son problemas exclusivos del ámbito sanitario si no que han pasado a ser responsabilidad de todos, de la familia, las instituciones educativas y la comunidad de aprendizaje del niño y la niña.

Cuando se considera la salud como el completo estado de bienestar físico, psíquico y social, tal como ha establecido la Organización Mundial para la Salud, se hace necesaria la evaluación del medio social en el que se desenvuelven los niños y niñas. Desde esa perspectiva, la salud es bastante compleja, mucho que el estado individual de cada uno de los miembros de una sociedad.

Las instituciones educativas tienen la obligación de adaptarse a las circunstancias, inquietudes y problemas de la sociedad en las que están insertas. En los últimos años se ha vuelto más notoria la importancia de las actividades físicas y el deporte en la educación para la práctica de las mismas, convirtiéndose en parte de los problemas que atiende la Salud Pública y su prevalencia está aumentando en las poblaciones infantiles en todo el mundo. Las escuelas, colegios, universidades y dentro de ellas, los profesores de Educación Física no pueden hacer caso omiso a esta problemática.

De acuerdo al ICSSPE, (2010) la Educación Física y el deporte en la escuela se considera el medio más eficaz e incluso para que todos los niños y niñas puedan adquirir y aprender las habilidades, el conocimiento y los valores de su participación a lo largo de sus vidas, con independencia de su capacidad, sexo, edad, cultura, raza/etnia, religión o nivel social. Educación Física y deporte en una institución educativa, es una materia que no conoce límites en el aula, sino que los trasciende al patio de la escuela, a los niños y niñas en juegos diversos independientes de la escuela e incluso puede llegar a estar presentes en actividades que desarrollen de adultos, es más no se limita a los niños y niñas, en oportunidades es practicada por toda la comunidad educativa. De ahí radica la importancia del presente que pretende determinar el alcance de esta materia en la vida de los niños y niñas, y aprovechar dicho alcance para la promoción de la salud de los mismos. 
Muy a pesar de los numerosos beneficios que trae consigo la práctica de actividad física y el deporte en niños y niñas, existe cierto grado elevado de preocupación por la inactividad física de niños, jóvenes y adultos. Esta preocupación crece, no solo a nivel nacional sino a nivel global y es debido a varios factores, tales como la existencia de cada vez un número mayor de niños con sobrepeso, obesidad o con altos riesgo de adquirirlos; de esto se derivan varias consecuencias puesto que la inactividad física es también uno de los factores de riesgo de enfermedades.

\section{METODOLOGÍA}

Esta investigación corresponde al tipo descriptiva - correlacional de enfoque cualicuantitativo, puesto que fueron recolectados datos y componentes de la incidencia de la Educación Física en niños y niñas, posteriormente estos datos fueron analizados de forma a poder caracterizarlos cualitativa y cuantitativamente.

La investigación descriptiva - correlacional busca especificar propiedades, características y rasgos importantes de cualquier fenómeno que se analice, midiendo de manera independiente los conceptos variables a lo que se refiere.

El tipo de estudio es descriptivo, porque a través del mismo se determinan las situaciones inherentes a las características del proceso de enseñanza-aprendizaje de la Educación Física y el Deporte.

El tipo de estudio descriptivo según, Tamayo y Tamayo (2003) propone describir de modo sistemático las características de una población, situación o área de interés.

En el presente trabajo se busca conocer la incidencia de la práctica del deporte sobre la práctica de hábitos saludables y valores, esto es correlación.

La investigación planteada describe y examina la realidad de hechos, presentando y describiendo las variables en el marco conceptual y operacional.

Asimismo, se realiza un estudio correlacional que determina si dos variables están correlacionadas o no. Esto significa analizar si un aumento o disminución en una variable coincide con un aumento o disminución en la otra variable.

En el desarrollo de la investigación se recurrió al enfoque cualitativo y cuantitativo, porque permitió definir correctamente al problema, planteada la hipótesis y aceptada, permite una relación funcional entre las variables para el análisis de la información.

El enfoque cuali-cuantitativo utiliza la relación y el análisis de datos para contestar preguntas de investigación y probar hipótesis previamente hechas, basándose en análisis estáticos y característicos sobre el fenómeno en busca de explicarlos, el propósito del 
enfoque cualitativo consiste en reconstruir la realidad, tal y como la observan los actores de un sistema social previamente definido. (Hernández, 2003).

Se empleó este método porque permitió conocer las opiniones de los docentes de la disciplina de Educación Física, de la directora y la evaluadora de la institución estudiada respecto al proceso de enseñanza aprendizaje.

Sampieri et.al (2002) definen al método cuantitativo como aquel que usa la recolección de datos para probar hipótesis con la base en la medición numérica y el análisis estadístico para establecer patrones de comportamiento.

A través del método cuantitativo se busca que en la investigación se ordenen los datos obtenidos de las diferentes técnicas de recolección de datos, las cuales se cuantificaron para conocer las características del proceso de enseñanza-aprendizaje de la asignatura Educación Física y de la conducta de los alumnos y de esta manera a través de los resultados se aprobará o refutará la hipótesis planteada.

Las mismas están constituidas por los alumnos y docentes de la disciplina de Educación Física, la directora y la evaluadora de la institución estudiada.

Las informaciones se obtuvieron de tesinas de grado, artículos científicos, revistas, libros de varios actores relacionados al tema y sitios en internet, que servirán como soporte para la presente investigación.

Los datos fueron colectados a través de: La encuesta a través de cuestionarios impresos, dadas a completar a los directivos, profesores de educación física y alumnos de la escuela, complementando a ello las observaciones a realizar por la responsable de esta investigación.

Encuesta: Se utilizó para recolectar los datos que interesan a la investigación. La misma fueron aplicada a los sujetos de estudio que conforman la muestra de la investigación.

Para este instrumento de recolección de datos se utilizó el cuestionario ya que de esa manera los encuestados pudieron contestar las preguntas por su propia cuenta. Los tipos de cuestionarios utilizados fueron el abierto y el cerrado.

La entrevista a partir de un Cuestionario Abierto: Se empleó este instrumento ya que permitió que los alumnos y docentes puedan mencionar sus opiniones, sentimientos y/o experiencias de las preguntas planteadas.

Cuestionario Cerrado: Se utilizó este instrumento ya que permitió recolectar informaciones específicas de los sujetos de estudio. 
Entrevista Semi-estructurada: Se utilizó la técnica de la entrevista semi-estrucurada, que permitió entablar una conversación directa con el sujeto de estudio facilitando obtener informaciones oportunas en el momento de aplicar el instrumento.

Se combinaron dos tipos de entrevista: la estructurada y la no estructurada, se recurrió a este instrumento para recabar informaciones directas de la Directora y la Evaluadora, este instrumento de recolección de datos se aplicó en forma intencionada

La muestra a considerar abarcó un total de 220 niños y niñas, alumnos del $1^{\circ}$ al $6^{\circ}$ grado de la Escuela Básica N579 Defensores del Chaco del Barrio San Lorenzo, se estudiarán estas edades a modo de delimitar infantes con características similares, evitando rangos muy extrapolado de edades; como los docentes, evaluadora y directora de la citada institución. Se utilizó el método aleatorio simple para elegir los alumnos.

\section{ANÁLISIS Y DISCUSIÓN}

Los resultados son presentados respecto a los objetivos propuestos en el trabajo.

1.1. Identificar las estrategias de intervención en la escuela para la promoción de la salud de los niños y niñas.

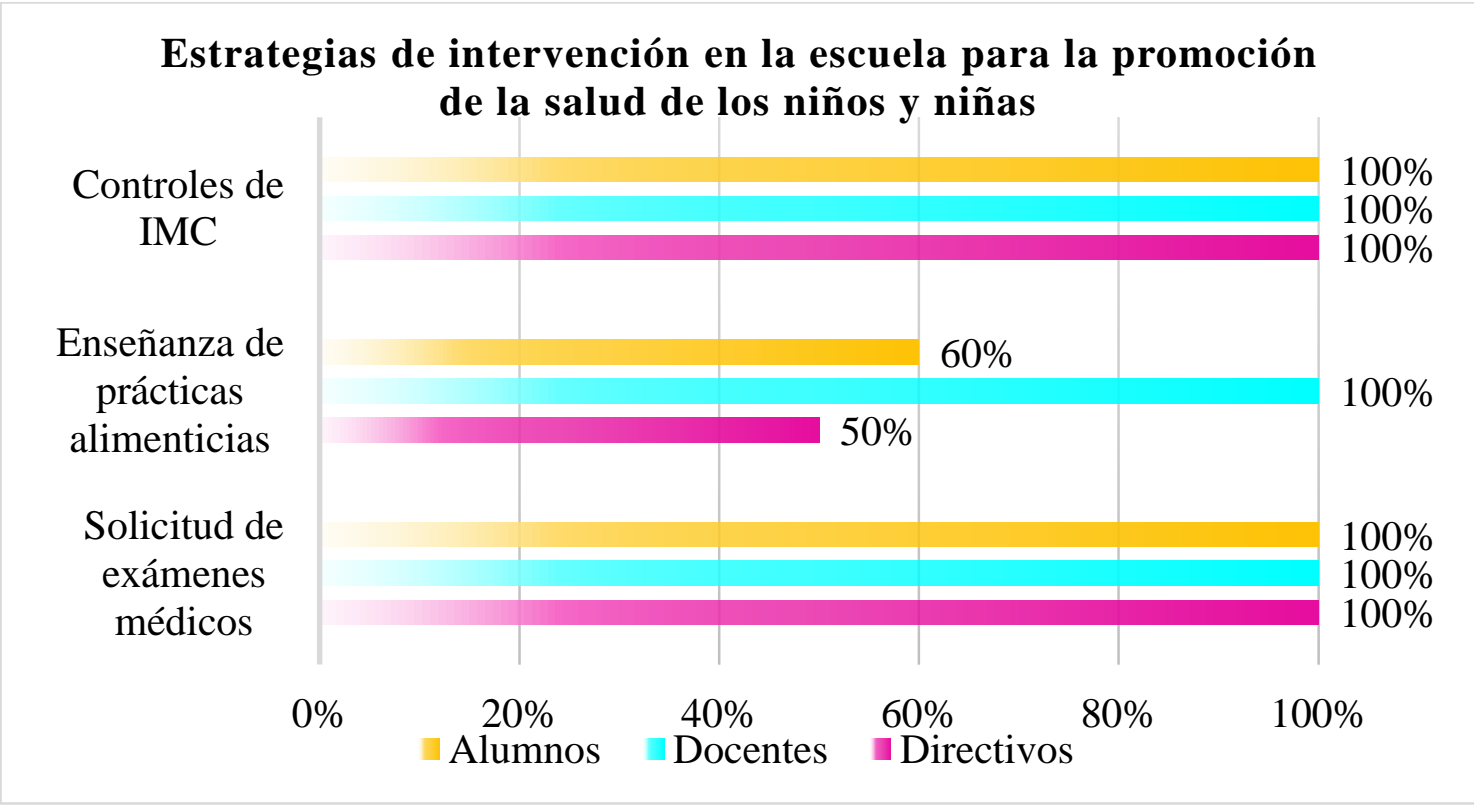

La medición de peso y altura para determinar el índice de masa corporal, según los docentes, es una estrategia útil para el cuidado de la salud de los alumnos, ayuda a alertarlos de posibles trastornos alimenticios. Los directivos afirman que la solicitud de exámenes médicos no solamente ayuda a la correcta práctica de deportes si no que previene enfermedades detectables desde muy temprana edad. 
Los alumnos consideran que los exámenes médicos solicitados por la institución, son una oportunidad para visitar al médico y conocer cómo se encuentran en cuanto a su salud, afirman también que su profesor de educación física y docente de grado, consideran que deben tener en cuenta en todo momento la hidratación contante del organismo a través de líquidos, como agua, jugos y otros.

El deporte y la actividad física son actividades culturales populares entre los niños y jóvenes y pueden, por consiguiente, servir para favorecer o enseñar determinadas habilidades para la vida (Escartí, Pascual y Gutiérrez, 2005). Dentro del ámbito educativo, conseguir conductas pro-sociales en los niños constituye uno de los objetivos prioritarios (Muñoz, Carreras y Braza, 2004), siendo los problemas de conducta un aspecto común en todas las áreas del currículum (Ishee, 2004).

En relación a las conductas apropiadas, la Real Academia Española (2001) las define como la "manera con que las personas se comportan en su vida, aplicando a cada cosa lo que le es propio o más conveniente". Por el contrario, las conductas disruptivas son "la música de fondo de la mayoría de nuestras aulas" (Torrego y Moreno, 2003, p. 129). De acuerdo con Mateo (2014), el trastorno de conducta suele manifestarse en niños y niñas cuyo manejo de comportamiento no ha sido el apropiado, en niños/as cuyos padres han sido excesivamente permisivos, y también es posible que aparezca un trastorno de conducta en el contexto escolar, sobre todo cuando el profesor/a no marca límites claros respecto a lo que está permitido hacer y lo que no.

De acuerdo con la teoría de la evaluación cognitiva de Deci y Ryan (1985) la conducta del alumnado está regulada por razones intrínsecas que producen placer, satisfacción o excitación mientras la persona realiza una actividad; razones configuradas por los valores y metas personales, determinadas por presiones internas orientadas a actuar de cara a evitar una evaluación negativa, como puede ser la culpabilidad, la vergüenza o la turbación; y por razones externas, referidas a conductas formadas por refuerzos externos, cumplimiento de reglas, o miedo al castigo.

Por otra parte, investigaciones previas han revelado que los profesores/as que promueven la orientación a la tarea en los alumnos y alumnas ayudan a adoptar mayores razones autodeterminadas para ser disciplinados (Barreda y Gómez, 2012). Los resultados han sido corroborados por Cervelló, Jiménez, Villar, Ramos y Santos-Rosa (2004) que encontraron además dos tipos diferentes de estrategias empleadas por los profesores para controlar el comportamiento de la clase. La primera de ellas hace referencia a las 
estrategias basadas en la idea de que el profesor puede mantener el orden mediante el uso de recompensas apropiadas al comportamiento disciplinado y mediante el uso de castigos en el caso de conductas indisciplinadas. La segunda categoría hace referencia a las estrategias consistentes en ayudar a los alumnos a ser responsables de su propio comportamiento, consiguiendo así incrementar su auto-determinación.

Ha sido demostrado que los comportamientos sociales apropiados mejoran cuando se implementan intervenciones para favorecer dichos comportamientos en el programa de educación física (Samalot-Rivera y Porretta, 2012; Madrid López, Prieto-Ayuso, Samalot-Rivera y Gil Madrona, en prensa; Monjas Aguado, Ponce Garzarán y Gea Fernández, 2015; Vidoni y Ward, 2009). En este sentido, es interesante destacar la existencia de modelos enfocados a la mejora de estas habilidades. Por ejemplo, el Modelo de responsabilidad social de Hellison (1995) que propone cinco niveles de desarrollo del alumnado: irresponsabilidad, autocontrol, implicación, responsabilidad e interés.

\subsection{Caracterizar los modelos de enseñanza que se utilizan en Educación Física} y el deporte para motivar a los niños y niñas para la iniciación deportiva.

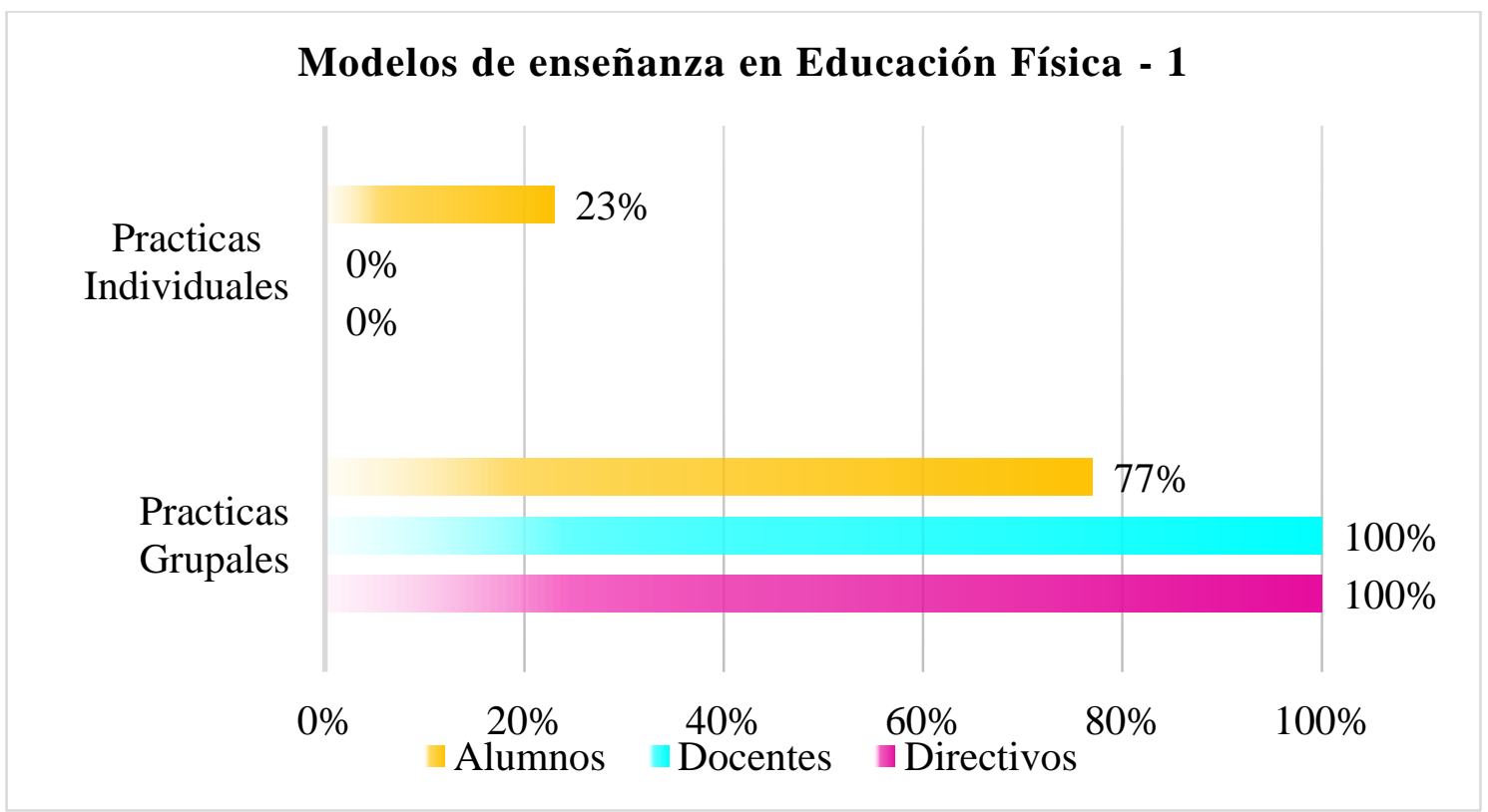

El docente encargado del área de Educación Física, directivos y profesor de grado, afirman que las prácticas de ejercicios grupales son las principales formas de iniciación deportiva, puesto que los niños y niñas buscan relacionarse entre sí y esto les impulsa a formar equipos para realizar pequeñas competencias, esta situación, le permite al docente de educación física controlar la correcta realización de los ejercicios y al mismo tiempo la evaluación continua y procesual. 
El 23\% de los niños y niñas manifestaron que las prácticas individuales los motivan a la realización de actividades físicas.

La educación física de calidad debe comprender la categoría de elementos educacionales como lo son el currículo, métodos de formación y evaluación de la educación física. También requiere del desarrollo de elementos de apoyo en el personal calificado, tiempo suficiente para la educación física en el horario, ambiente e instalaciones seguras y apropiadas para que un buen programa pueda desarrollarse (Lopez de D’Amico; Ya Ho; Antola; Benn; Dinold y Holzweg, 2018)

Los procesos de formación que se están siendo desarrollados en la clase de educación física en las instituciones educativas, deben poseen mayor relevancia en los escenarios educativos, ya que la clase se convierte en un espacio determinado por la necesidad de controlar, limitar, disciplinar y realizar acciones que no se alejan de los propósitos educativos que son promulgados en la legislación educativa, y por aquellos emanados en los proyectos educativos (Betancur; López y Rodríguez, 2018).

Para producir una educación física de calidad es necesario tener en cuenta un buen ambiente escolar que facilite la integración entre niños y niñas.

Los ambientes escolares que generan aprendizaje, facilitan canales de comunicación bidireccional donde los estudiantes no son los únicos receptores, el maestro escucha y toma en consideración las sugerencias de sus estudiantes, a la vez que estos escuchan las anotaciones y percepciones de los demás integrantes de los grupos (Arias, 2018).

La propuesta de trabajo por equipos enriquece el ambiente del aula, moviliza los conocimientos y facilita el intercambio, además de la posibilidad de explorar diversas estrategias didácticas para la construcción de conocimiento. Los ambientes escolares de aprendizaje, trascienden el espacio físico del aula, cualquier escenario es propicio para la indagación, el conocimiento y la socialización, mientras el maestro tenga una intención clara de enseñanza y un objetivo concreto en su proceso pedagógico (Arias, 2018). 


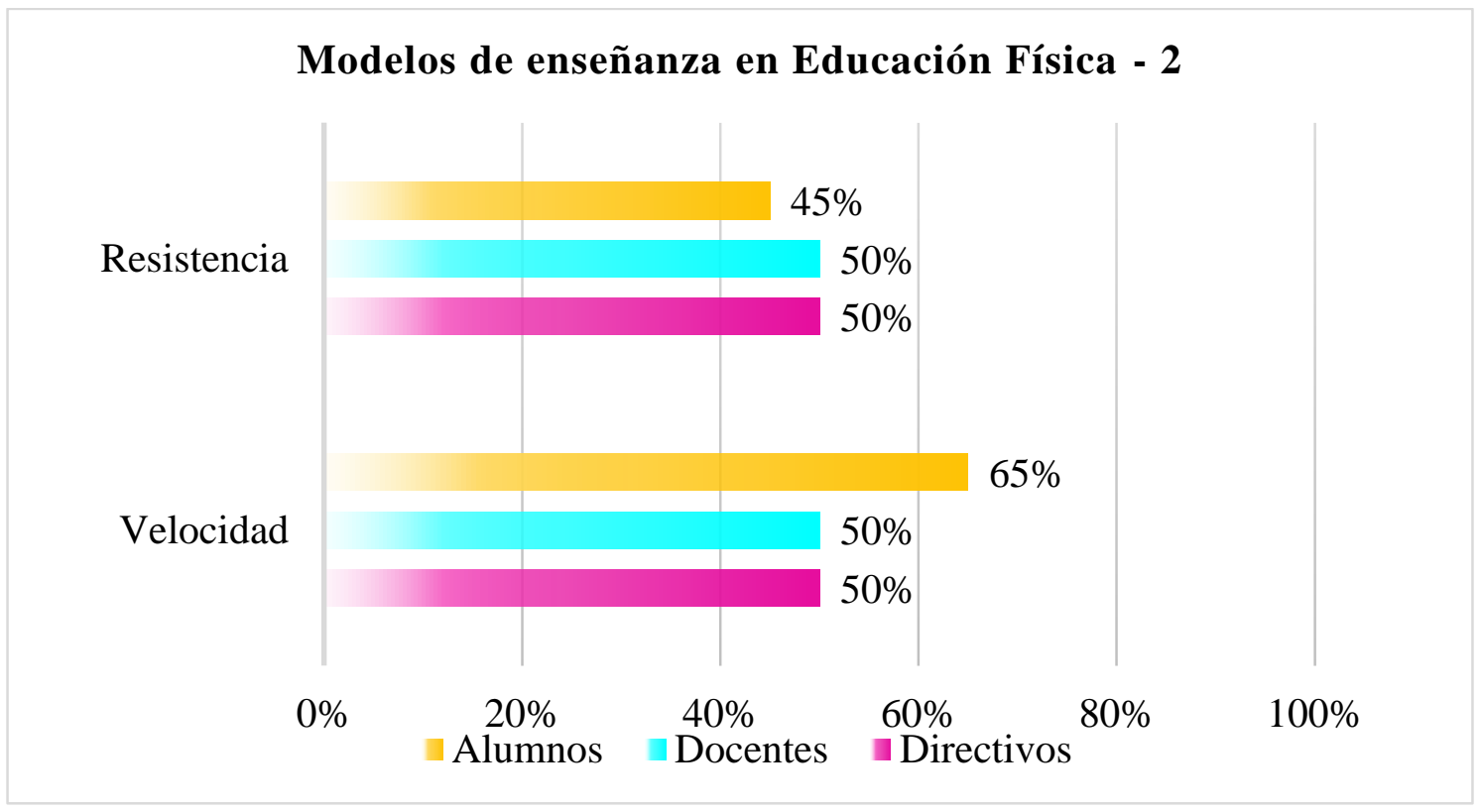

En cuanto a la práctica de actividades aeróbicas (presencia de oxígeno. Ejemplos: nadar, bailar, caminar), o anaeróbicas (ausencia de oxígeno. Ejemplo: correr series muy intensas), donde el metabolismo se desarrolla exclusivamente en los músculos y sus reservas de energía, sin usar el oxígeno de la respiración, en todos los grados del $1^{\circ}$ al $6^{\circ}$ grado el profesor de educación física, desarrolla en sus contenidos programáticos las capacidades aeróbicas, como diferentes clases de juegos recreativos, ejercicios individuales y colectivos o grupales.

Así también el organismo necesita prepararse progresivamente para las actividades anaeróbicas de la educación física.

Desde la infancia el niño se construye a partir de las experiencias y aprendizajes que le significaron un roce emotivo y sensitivo. Eso hace notar que los seres humanos todo el tiempo están observando, vivenciando, percibiendo y por ende fortaleciendo su personalidad; por eso no es indiferente a la escuela la consigna de integrar en un espacio de socialización contenidos formativo para la vida (Padilla, Dávila, Torres, Escalante, y González, 2008).

La niñez es considerada una etapa clave en la adquisición del estilo de vida, en la que se enfrenta a diversas conductas y experiencias, resultado de sus relaciones y de la acción de los agentes de socialización, que tendrán una importancia primordial en la formación de los hábitos de conductas saludables y positivas que tengan continuidad durante toda su vida. 
En la actualidad, deja entrever la necesidad de nutrir la educación con los talentos y habilidades de los estudiantes. Al potenciar sus capacidades se estimula su desarrollo emocional, social y cognitivo, de esta manera, la escuela podría convertirse en el real escenario que educa para la vida. Tal como Chaparro lo asevera:

(...) El ambiente educativo no se limita a las condiciones materiales necesarias para la implementación del currículo, cualquiera que sea su concepción, o a las relaciones interpersonales básicas entre maestros y alumnos. Por el contrario, se instaura en las dinámicas que constituyen los procesos educativos y que involucran acciones, experiencias y vivencias por cada uno de los participantes; actitudes, condiciones materiales y socio afectivas, múltiples relaciones con el entorno y la infraestructura necesaria para la concreción de los propósitos culturales que se hacen explícitos en toda propuesta educativa (Chaparro, 1995: 2, citado por Arias, 2018).

Conviene subrayar la necesidad, no solo de espacios físicos de agradabilidad y conexión sensorial, para el acto pedagógico, sino de la interconexión que surge en las relaciones y actos comunicativos dentro de ese espacio para obtener como resultado un ambiente escolar propicio para la convivencia y la construcción de conocimiento académico (Arias, 2018). Además, de la oportunidad de desarrollar habilidades de metacognición basados en procesos activos y dinámicos desde la experiencia real como lo demuestran las tendencias del constructivismo propuestas por Piaget (1952, citado por Arias, 2018).

\subsection{Describir las características de la conducta aprendida como los hábitos} saludables, valores y normas aprendidas en la Educación Física y el deporte por los niños y niñas

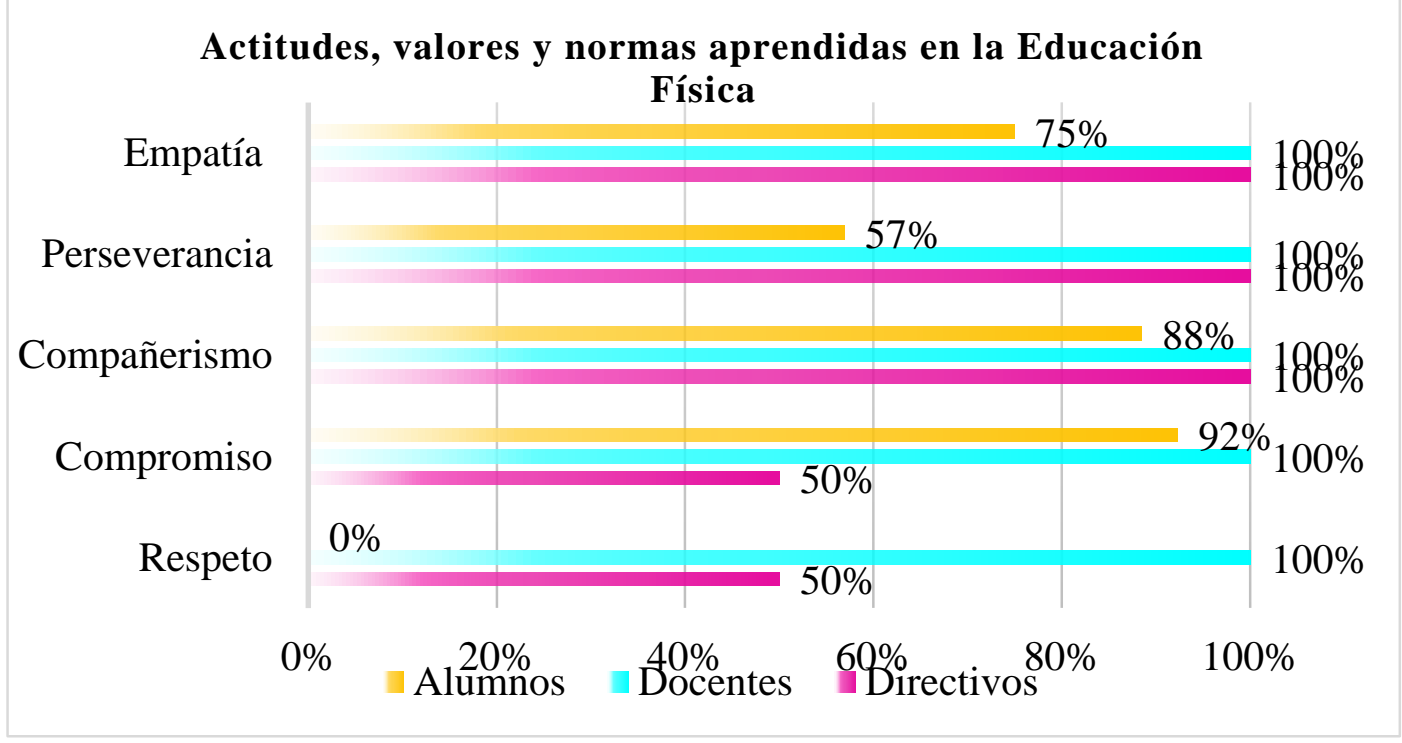


Para los directivos la empatía, perseverancia y compañerismos son enseñados y aprendidos, el $50 \%$ de los directivos también cita al respeto y compromiso como inculcados en la institución en la materia de Educación Física.

Los docentes afirman la totalidad de los valores citados y añaden la autovaloración que tratan de trasmitir a los niños y niñas desde edades iniciales y aún más en el segundo ciclo. Los alumnos resaltan el compañerismo, compromiso y empatía.

En todos estos aspectos muy ligados al compromiso de la materia, los alumnos mencionaron que además adquirieron hábitos y normas para su independencia personal y social, como también tener en cuenta la disciplina, el buen comportamiento, el orden y la limpieza en cuanto a lo personal e institucional, en todo lugar y en todo momento.

Las funciones cognoscitivas son esenciales para enfrentarse con éxito a las demandas que trae consigo el aprendizaje. Desde su nacimiento, cada individuo aprende de los sucesos cotidianos que lo rodean, pero no es hasta que ingresa en la escuela donde el aprendizaje se vuelve formal y, la adquisición de la lectura y la escritura, así como los conocimientos básicos sobre matemáticas y ciencias, requieren de atender, comparar, diferenciar y buscar semejanzas para lograr asociar e integrar las nuevas ideas con las ya existentes (Castillo Parra, Gómez y Ostrosky-Solís, 2009, citado por González, 2016).

Las habilidades cognitivas incluyen tanto las habilidades cognitivas básicas, tales como la función ejecutiva, la atención, la memoria, la comprensión verbal y el procesamiento de la información, así como las actitudes y creencias que influyen en el rendimiento académico, como la motivación, el autoconcepto, la satisfacción y el entorno escolar (González, 2016).

Dentro del ámbito de la Educación Física, se ha puesto de manifiesto que los comportamientos deseados en Educación Física están dentro del amplio concepto denominado "Juego limpio" (Vidoni y Ward 2006), definido por Contreras (2013) como los particulares comportamientos caracterizados por el principio de justicia para todos, en los cuales no hay lugar a la tentativa de ganar una ventaja injusta sobre el adversario ni intencional ni fortuitamente.

La mejor estrategia como lo menciona Fabián Sanabria, es la educación, la cual se debe llevar a cabo con el objetivo de contribuir a la construcción de una mejor sociedad, reduciendo la presencia de actos violentos y agresiones, pero para esto primero se requiere profundizar qué se entiende por violencia, según la Organización Mundial de la Salud: violencia es el uso deliberado de la fuerza física o el poder como amenaza o de manera 
contra uno mismo, otra persona, grupo o comunidad que cause o tenga posibilidades de causar lesiones, muerte, daños psicológicos, trastornos del desarrollo o privaciones; implica el usos de amenazas de violencia física o psicológica, con intención de hacer daño de manera recurrente y como forma de resolver conflictos, no es innata a los seres humanos, es aprendizaje, una realidad histórica, humana y socialmente aprendida (O.M.S, 2015, p.1).

La O.M.S ha hecho un análisis previo de muchos países y entornos para llegar a comprender que la violencia tiene algo que es clave, y es que la violencia no es algo innato de los seres humanos, es un aprendizaje previo que se realiza en el transcurso de nuestra formación, esa formación de principios y valores morales, que se aprende por medio de la observación que se nos enseñan en el núcleo familiar, y en la escuela en las primeras edades.

A partir de esto se entiende que la violencia escolar es la violencia que se manifiesta en el contexto educativo formal, la cual ha existido desde los mismos momentos de la aparición de la escuela, siendo en otro momentos menos visible por diversas circunstancias, por el poder y la violencia que empleaban los maestros, con modelos tradicionales de educación, pero a través del tiempo se han generado cambios, con el paso de la modernización educativa, al estudiante se le permitió más libertad y reconocimiento en el proceso de enseñanza- aprendizaje, pero se cayó en la laxitud y de la libertad se pasó al libertinaje dejando en ocasiones a padres, maestros y Estado sin herramientas para el control de niños-niñas y adolescentes. Al respeto Morales Néstor resalta que:

Se victimizó tanto esta población que de un momento a otro pasaron a ser victimarios también, se enfatizó en el trabajo, promulgación y búsqueda de protegerlos y reconocer sus derechos, pero se olvidó que también son agentes activos en un proceso social y que tienen responsabilidades de acuerdo a sus edades cronológicas (Morales, 2015 p 10).

Hoy en día algo sucede con nuestros jóvenes ya que cuentan con conductas agresivas notorias como gritos, falta de cumplimiento de reglas, agresión física, por lo cual se considera se está fallando en algunos aspectos educativos, por eso es importante desde lo mencionado buscar estrategias que permitan entender el estudiante que demanda el siglo XXI, de allí la importancia que este trabajo le da al abordaje del: respeto, la conducta agresiva, la comunicación asertiva, y su relación en el análisis del contexto.

En esta medida, para Kant el respeto es un valor moral que nos permite reconocer, aceptar y considerar las cualidades del individuo y sus derechos: 
Los individuos deben ser respetados porque son fines en sí mismos y no medios para los fines de otros, al ser un fin en sí mismos poseen un valor intrínseco y absoluto en el sentido de que no puede ser devaluado o ignorado por otros criterios, por este motivo es que los individuos tenemos este valor tan especial llamado por Kant la dignidad, que otros filósofos han denominado como valor inherente. (Kant, 1956)

Esta definición que nos lleva a pensar que cada ser humano es único y libre, lleva a pensar que no se puede hablar de respeto sin comprender que es la dignidad, esa misma que nos permite ser respetados ya que ocupamos un espacio en el universo, que no se puede ser esclavo de ninguna otra persona o movimiento cultural, por esto se debe educar con estrategias y didácticas, que siguiendo lo propuesto por Fabián Sanabria, podrían ser escuchar y comprender, profundizando para ello en el manejo de la comunicación asertiva con el propósito de una sana convivencia y unas mejores relaciones interpersonales.

Es propicio mencionar que la consecuencia de la falta de respeto en muchos casos es la violencia, esa que tanto dolor ha causado en un país como Colombia; Por eso se hace necesario evidenciar cuales son esos factores que hacen que el niño sea violento, identificar como el contexto sociocultural afecta la formación para la convivencia; es ahí donde pretende incidir el proyecto partiendo de entender que la agresividad es un tema complejo, que muchos autores como: Aaron T, Skiner, Bandura, Maslow, Freud, la identifican como una conducta que está presente en nuestra sociedad, tanto a nivel familiar, laboral, entre iguales, a nivel nacional y mundial.

Dentro de los muchos estudiosos que se preocuparon por la conducta humana, y fundamentalmente por el comportamiento agresivo de los seres humanos, Freud quien es considerado el padre del psicoanálisis escudriñó las profundidades de la psique, ese mundo misterioso y controvertido de los seres humanos, y estableció que las conductas agresivas son:

Fenómenos que se producen como resultado de las múltiples manifestaciones, acciones y reacciones del hombre, que se originaban de los instintos humanos; algunas de las principales manifestaciones de la agresividad se dan a raíz de que es una conducta de defensa y se manifiestan de manera psicológica, verbales, y física. (Sigmund Freud, 1940).

Según lo mencionado por Freud la agresión es como un vaso que con cada frustración se va llenando de agua, así entonces, llegará un momento en el que el vaso estará lleno y 
rebalsado de agua, así funciona la frustración que al rebalsar se transforma en agresión, es así que funciona nuestro ser interior respecto a la agresión.

Ahora bien, para Freud, la agresión es una represión que en algún momento un detonante hace que se active y explote afectando al otro; en consecuencia, a partir del análisis del contexto se percibe que los niños y niñas identifican modelos a seguir que lo va formando $\mathrm{y}$ le permiten generar un autocontrol frente a las frustraciones y agresiones que se presentan en su cotidianidad.

Es pertinente resaltar que dichos modelos pueden ser buenos o malos, por ejemplo: un padre que maltrata a su esposa delante de sus hijos, con qué carácter va a corregir a su hijo cuando este golpea a un compañero; si es un mal modelo, por ello el propósito es incidir en la toma de decisión frente a la acción de agresividad que se le presenta al sujeto. El psicólogo Eduardo Hernández; describe que: la agresividad se manifiesta de varias formas, y que estas manifestaciones van por supuesto en relación a los contextos, no es lo mismo la reacción de un niño frente a sus padres que frente a sus amigos, y muchos menos en un salón de clases que en un espacio público (2008, p3).

Ya se había identificado que la agresión cuenta con dos pilares característicos de manifestación que son la física y la verbal, es así que la agresión verbal se entiende como: Toda agresión que no es física, también se traduce como agresión emocional, ya que son palabras que afectan negativamente la situación emocional de la víctima, es la más silenciosa, la que menos lleva a la denuncia a nivel judicial, la que incluso se admite socialmente y la que, no por eso, se sufre menos (Hernández, E. 2008 p3).

Esta manifestación es muy notoria en todo contexto, familiar, escolar, público, privado, ya que se crean registros lingüísticos que como lo menciona el autor generan siempre una víctima, la cual puede enfrentarse a casos que lo llevarán en el peor de las situaciones a daños irreparables, pero no es ajena a la siguiente manifestación o conducta, la agresión física, que se trata de una conducta algo fuerte, ya que la afectación física puede llegar a generar que las víctimas sufran alguna discapacidad o limitación que le impida seguir con su vida cotidiana normal, es por esto que se plantea un proyecto cuyo propósito es educar para que disminuyan las agresiones en todas sus manifestaciones. Se sabe que no es fácil convivir en un país tan violento, pero se tienen que incentivar a las futuras generaciones a reflexionar las acciones, para llegar a que el ser humano, pueda transformarse en busca de las conductas menos agresivas. 
Teniendo presente que las conductas se consideran como el modo de actuar de un individuo, observable, medible y modificable, a continuación, se presentan unas características que nos permiten evidenciar si un individuo tiende a generar una conducta agresiva, estas son:

Accesos de cólera, actos de desobediencia ante la autoridad y las normas del entorno, amenazas verbales, daños a cosas materiales, deterioro en la actividad social y académica por episodios de rabia, gritos, molestar a otros sin justa razón, mostrarse iracundo o resentido, pleitos (Hernández, E. 2008 p4).

Estas características deben presentarse de manera frecuente, con cierta intensidad y duración adecuada para pensarse que ya se constituyen como patrón conductual, ante esto existe un elemento clave y es la comunicación ya que esta permite expresar ideas sentimientos y todas aquellas emociones que nos invaden de manera verbal y física. Por eso es propicio mencionar que el trabajo toma como herramienta la comunicación asertiva, para aportar a la solución de la problemática empleando como estrategia la interacción en la clase de educación física.

\section{CONCLUSIÓN}

La Organización Mundial de la Salud considera la actividad física como uno de los principales factores que intervienen en el estado de salud de las personas y la principal estrategia en la prevención de la obesidad, la Educación Física en una institución educativa, es una materia que no conoce límites en el aula; sino que los trasciende al patio de la escuela, a los niños y niñas en juegos diversos independientes de la escuela e incluso puede llegar a estar presentes en actividades inter escolares.

La práctica de deporte tiene una larga historia como actividad que se utilizaba para la actividad de recreación. Debido a las transformaciones de la sociedad y el desarrollo social y económico se comenzó a comprender el aspecto del deporte en algunos lugares educación física, en la educación y en la era moderna comenzó a tener un nuevo enfoque (Lopez de D’Amico; Ya Ho; Antola; Benn; Dinold y Holzweg, 2018).

La comprensión actual acerca del deporte escolar, cambió la concepción. Se utilizaron diversos enfoques a objeto de mejorar el proceso de enseñanza-aprendizaje por parte del alumno. Conceptos como 'educación a través de la actividad física', 'concepto de vida', 'educación para toda la vida', 'aprendiendo a aprender en deporte', 'igualdad en el aprendizaje en el deporte', 'aprendizaje a través del juego', 'concepto del juego', 
‘enseñando juegos para la comprensión', entre otras, florecieron en el campo educacional. (Lopez de D’Amico; Ya Ho; Antola; Benn; Dinold y Holzweg, 2018)

A pesar de los diversos conceptos que se le pueda atribuir, la práctica de la actividad física posee un gran número de beneficios fisiológicos y psicológicos no solo para el niño, sino toda persona que la práctica de manera regular; como así también las conductas sedentarias y los hábitos alimentarios inadecuados pueden representar una carga para la salud pública.

La educación física en la escuela posee un rol esencial para la promoción de la actividad física, las preferencias de las actividades físicas ya dependerán de cada niño y niña, pero todas ellas los estarán beneficiando.

La familia constituye un papel fundamental en la adquisición de unos adecuados hábitos saludables, sin descartar el rol que desempeñan los profesores desde el ámbito escolar, en la formación y configuración de estilos de vidas saludables.

Para la comprensión de procesos motivacionales, determinante en las conductas saludables de los niños y niñas, ha sido la teoría social-cognitiva, el modelo cognitivosocial está construido sobre expectativas y valores que los alumnos adjudican a diferentes metas y actividades de ejecución.

La actividad física no se limita a los niños y niñas, en oportunidades es practicada por toda la comunidad educativa. De ahí radica la incidencia de la educación física en la conducta de escolares, considerando por sobre todas las cosas la promoción de la salud, donde aprenden hábitos saludables para su formación integral.

Las prácticas grupales son preferidas a las individuales porque se apela a la necesidad de comunicarse y compartir de los niños y niñas, según lo manifiesto por los docentes y directivos. Un grupo minoritario de alumnos prefiere las actividades individuales implementadas por el docente de Educación Física.

La Escuela Básica N578 Defensores del Chaco del Barrio San Lorenzo promueve no solo la práctica de deportes en la materia de Educación Física, según los directivos, docentes y alumnos, los valores de empatía, compañerismo y compromiso forman parte de la educación recibida.

Los beneficios de la actividad física (AF) sobre niños y jóvenes están ampliamente demostrados. En la actualidad el ejercicio físico resulta fundamental en el proceso educativo de escolares, tanto para el desarrollo de valores sociales e individuales como para el fomento de hábitos saludables que hagan frente a los actuales problemas de salud 
pública presentes en la infancia y adolescencia y entre los que destacan los preocupantes hábitos sedentarios y la obesidad (Reloba, Chirosa y Reigal, 2016).

Las instituciones educativas tienen la obligación de adaptarse a las circunstancias, inquietudes y problemas de la sociedad en las que están insertas. En los últimos años se ha vuelto más notoria la importancia de las actividades físicas y de la educación en hábitos para la práctica de las mismas, convirtiéndose en parte de los problemas que atiende la Salud Pública y su prevalencia está aumentando en las poblaciones infantiles en todo el mundo. Las escuelas, colegios, universidades, lo que les compete a los profesores de Educación Física, no pueden hacer caso omiso a esta problemática.

Así también la Organización Mundial de la Salud (2015) considera la actividad física (AF) como uno de los principales factores que intervienen en el estado de salud de las personas y la principal estrategia en la prevención de la obesidad.

Cuando se considera la salud como el completo estado de bienestar físico, psíquico y social, tal como ha establecido la Organización Mundial para la Salud, se hace necesaria la evaluación e intervención de toda la comunidad educativa, para garantizar y facilitar desde el inicio de las actividades, el trabajo del docente de educación física, del maestro de grado y directivo; teniendo en cuenta siempre la participación de toda la comunidad educativa.

Es muy importante que los niños y niñas realizan actividad física desde muy temprana edad para adquirir habilidades motrices básicas y contar con el certificado médico a comienzo del año, para facilitar el trabajo del profesor de educación física y que todo esto se desarrolle en un ambiente escolar apropiado.

Los ambientes escolares subrayan la necesidad de ser revisados y analizados por los maestros como una forma de autoevaluar las practicas pedagógicas de forma introspectiva para marcar la ruta o el camino en la construcción de un aprendizaje significativo tal como lo plantea Novak (1988) pues todo acto educativo en un ambiente de aprendizaje, genera una acción que cambia los significados (pensar) y sentimientos, tanto del estudiante como del maestro.

En consecuencia, Cano y Lledó (1995, citado por Arias, 2018) piensa el aula como un lugar de encuentro donde el ambiente escolar se construye a partir de la posibilidad del acercamiento entre los integrantes del grupo, puesto que el intercambio creará relaciones de cohesión con quienes tienen metas u objetivos comunes. De esta manera, se toma una perspectiva de la socialización, el contacto con las proyecciones de otros individuos y la 
concreción de metas similares que lleva al estudiante hacia el análisis de otras realidades y contextos desde una óptica crítica, de crecimiento personal y solidario (Arias, 2018).

Otro aspecto necesario en la generación de ambientes escolares para la convivencia y el aprendizaje es la disposición y contacto con los materiales para el trabajo, la organización de las mesas y la ubicación espacial del aula, son elementos relevantes debido a su carácter integrador y participativo aspectos comunes en la propuesta metodológica del modelo pedagógico escuela nueva que propende el mejoramiento de la eficiencia y la calidad de la educación promoviendo procesos de aprendizaje activos, evaluación cualitativa y permanente, además del fortalecimiento de la relación escuela - comunidad (Arias, 2018).

En este contexto, la educación física, en su carácter didáctico-crítico, se propone la formación de un sujeto reflexivo y creativo dentro de una heterogeneidad disciplinaria de conocimientos y de formas de socialización que demandan definir la identidad educativa de esta disciplina, la que, por su propia naturaleza, está sujeta en mayor medida que ninguna otra materia curricular a la universalización y a los procesos de globalización.

Si se tiene en cuenta que las actividades físicas y los deportes son una materia de gran impacto social, educativo, político y económico, que cuenta con espacios propios en todos los medios de comunicación e información; que del buen o mal entendimiento de estas actividades en la práctica o aplicación personal depende la salud, la calidad y la esperanza de vida de los ciudadanos; y que fenómenos dependientes de una buena o mala educación física, son determinantes de situaciones de seguridad colectiva (Antúnez, 2017).

\section{LISTA DE REFERENCIAS}

Ajuriaguerra, J. (1975). Manual de psiquiatría infantil. España.

Antúnez Pérez, A; (2017). La evaluación de la educación física, los prototipos de enseñante y sus contextos Educere, Universidad de los Andes Mérida, Venezuela

Arias Vinasco; I (2018), Ambientes escolares: un espacio para el reconocimiento y respeto por la diversidad. Escuela Normal Superior del Quindío, Colombia. Universidad. La Gran Colombia.

Ato, M., López, J. J., y Benavente, A. (2013). Un sistema de clasificación de los diseños de investigación en psicología. Anales de psicología, 29(3), 1038-1059.

Aymerich, C. (1971). Expresión y arte en la escuela 1, España.Barreda, M. y Gómez. (2012). El docente como gestor del clima del aula. Factores a tener en cuenta. Trabajo de Máster: Universidad de Cantabria, Santander. 
Bass, B. M. (1983). Organizational decision making. Homewood, IL: Irwin.

Betancur-Agudelo, J; López-Ávila, C; Arcila-Rodríguez, W. (2018). El Docente de Educación Física y sus Prácticas Pedagógicas. Prácticas Pedagógicas. Revista Latinoamericana de Estudios Educativos (Colombia). Universidad de Caldas, Colombia Disponible en: http://www.redalyc.org/articulo.oa?id=134156702002.DOI: https://doi.org/10.17151/rlee.2018.14.1.2cionales (PEI).

Blández, J. (1994). Una propuesta didáctica centrada en la construcción de ambientes de aprendizaje. Actas del I Congreso Nacional de Educación Física de Facultades de Ciencias de la Educación y XII de Escuelas de Magisterio. Sevilla.

Bolívar, A. (2002). La evaluación de actitudes y valores: problemas y propuestas. En Castillo Arredondo, S. Compromisos de la evaluación Educativa, pp. 91-114. Pearson Educación/Prentice-Hall.

Cecchini, J. A., Fernández Losa, J., y González, C. (2008). Repercusiones del programa Delfos de educación en valores a través del deporte en jóvenes escolares. Revista de Educación, 346, 167-186

Devís y Peiró (1993). La actividad física y la promoción de la salud en niños/as y jóvenes: la escuela y la Educación Física

Dirección General de Estadísticas, Encuestas y Censos (2000). Proyección de la Población, Áreas Urbana y Rural por Sexo y Edad, 2000-2025.

Fernández, J. (2017). El Ciclo del Aprendizaje Cooperativo: una guía para implementar de manera efectiva el aprendizaje cooperativo en educación física. Retos, Nuevas Tendencias en Educación Física, Deporte y Recreación, 32, 264-269.

Fernández, J., Calderón, A., Pérez, A.y Hortiguela, D. (2016). Modelos pedagógicos en educación física: consideraciones teórico-prácticas para docentes. Revista española de Educación Física y deportes. 1133-6366 y ISSNe: 2387-161X. Número 413, año LXVIII, $2^{\circ}$ trimestre, España.

Fernández, J.y Méndez, A. (2016). El Aprendizaje Cooperativo: Modelo Pedagógico para Educación Física. Retos, Nuevas Tendencias en Educación Física, Deporte y Recreación, 29, 201-206.

Freud, Sigmund. (1940), Conductas agresivas, capitulo II, El esquema del psicoanálisis, Reino unido editorial, Worcester. 
González Hernández, J; y Portolés A, (2016). Recomendaciones de actividad física y su relación con el rendimiento académico en adolescentes de la Región de Murcia. Physical activity recommendations and their relation with academic performance in adolescents from the Region of . Federación Española - (España)

González, J. (2015). Análisis del autoconcepto en relación con factores educativos, familiares, físicos y psicosociales en adolescentes de la provincia de granada. Tesis doctoral. Universidad de Granada

Hamodi, C., López, V.y López, A. (2015). Medios, técnicas e instrumentos de evaluación formativa y compartida del aprendizaje en educación superior. Revista Perfiles Educativos. vol. XXXVII, núm. 147, 2015. IISUE-UNAM.

Hernández Sampieri, Roberto, Carlos FERNÁNDEZ COLLADO y Pilar BAPTISTA LUCIO (2003) Metodología de la Investigación. México, McGraw-Hill.

Hernández, Eduardo. (2008), Conductas agresivas en el entorno, Caracas, editorial, C.I.P.P.S.V.

International Position Statement on Physical Education (2010). International Council of Sport Science and Physical Education (ICSSPE). Recuperado de: http://www.icsspe.org/content/international-position-statement-physicaleducation

Larraz, A y Figueroa, J. (1988). El acondicionamiento de los patios de recreo. Revista Cuadernos de Pedagogía, No 159, pp. 24 -29.

López de D’Amico, R; Ya Ho, Walter King; Antola, Branislav; Benn, T; Dinold, M y Holzweg, Tansin. (2018). Compartiendo perspectivas globales acerca de la Educación Física de calidad. Educere, Universidad de los Andes

Mendiara, J. (1997). Educación Física y aprendizajes tempranos. Contribución al desarrollo global de los niños de 3 a 6 años y estudio de sus estrategias de aprendizaje en espacios de acción y aventura. Zaragoza.

Navarro, R.y Borja, M., (2018). Incidencia de los juegos cooperativos en el autoconcepto físico de escolares de educación primaria. Revista Federación Española de Asociaciones de Docentes de Educación Física (FEADEF). ISSN: Edición impresa: 1579-1726. Edición Web: 1988-2041 (www.retos.org). Universidad de Santiago de Compostela. España.

Navas, L.y Soriano, J. (2016). Análisis de los motivos para practicar o no actividades físicas extracurriculares y su relación con el autoconcepto físico en estudiantes 
chilenos. Revista Iberoamericana de Psicología del Ejercicio y el Deporte. Vol. 11(1), 69-76.

Ramos de Balazs, Argenira; López de D’Amico, Rosa; Guerrero de Hojas, Gladys (2015). Educación física y su relación con la salud en la formación integral. Experiencia desde el contexto educativo en Venezuela. Revista Iberoamericana de Psicología del Ejercicio y el Deporte, Universidad de Las Palmas de Gran Canaria. Las Palmas de Gran Canaria, España

Reloba, S.; Chirosa, L..; Reigal, R.. (2016). Relación entre actividad física, procesos cognitivos y rendimiento académico de escolares. Relation of physical activity, cognitive and academic performance in children: Review of current literature. Revisión de la literatura actual Revista Andaluza de Medicina del Deporte, Centro Andaluz de Medicina del Deporte Sevilla, España

Rodríguez, G; et al., (2016). Actividad física, ejercicio y deporte en la lucha contra la obesidad infantil y juvenil Nutrición Hospitalaria. Actividad física, ejercicio y deporte en la lucha contra la obesidad infantil y juvenil. Physical activity, exercise and sport practice to fi ght against youth and childhood obesity. Sociedad Española de Nutrición Parenteral y Enteral Madrid, España.

Samalot-Rivera A. y Porretta, D. (2012). The influence of social skills instruction on sport and game related behaviours of students with emotional or behavioural disorders, Physical Education \& Sport Pedagogy, DOI:10.1080/17408989.2011.631004.

Samalot-Rivera, A. (2013). Modelos a utilizar para la enseñanza de valores y destrezas sociales apropiadas en la Educación Física y el Deporte: una revisión de la literatura. Lecturas, Educación Física y Deportes, 184

Santos Guerra, M. (1993). La evaluación: un proceso de diálogo, comprensión y mejora. Málaga. Aljibe.

Siedentop, D., Hastie, P., y Van der Mars, H. (2004). Complete guide to sport education. Champaign, IL: Human Kinetics.

Torrego, J.C. y Moreno, J.M. (2003). Convivencia y disciplina en la escuela. El aprendizaje de la democracia. Madrid: Alianza.

Urquidez-Romero, R, et.al., (2017). Promoción de la actividad física en niños del norte de México: efectividad de una intervención educativa Acta Universitaria, vol. 27, 
núm. 2, marzo-abril, 2017, pp. 32-38 Universidad de Guanajuato Guanajuato, México

Velázquez, C (2015). Aprendizaje cooperativo en Educación Física: estado de la cuestión y propuesta de intervención. Revista Retos, Nuevas Tendencias en Educación Física, Deporte y Recreación, 28, 234-239.

Velázquez, C. (2015). Aprendizaje cooperativo en Educación Física: estado de la cuestión y propuesta de intervención. Retos, Nuevas Tendencias en Educación Física, Deporte y Recreación, 28, 234-239.

Vidoni. C., \& Ulman, J. D. (2010). Fair play instruction during middle school physical education: a systematic replication. Journal of Behavioral Health and Medicine, $1,127-136$.

Vidoni. C., \& Ward, P. (2006). Effects of a Dependent Group-oriented Contingency on Middle School Physical Education Students' Fair Play Behaviors. Journal of Behavioral Education, 15, 81-92. 